\title{
Truncal muscle tonus in progressive supranuclear palsy
}

\author{
Akiyo Tanigawa, Atsushi Komiyama, Osamu Hasegawa
}

\begin{abstract}
Objective-To elucidate the character and distribution of the abnormal muscle tonus in the body axis in progressive supranuclear palsy. Although neck hypertonus has been well described in progressive supranuclear palsy, little is known about the involvement of the truncal muscles.

Methods-Muscle tonus of the neck and trunk was separately investigated in 13 patients with progressive supranuclear palsy by clinical examination and surface EMG during passive rotation. Muscle hypertonus was graded according to a four point scale, and subjected to statistical analysis. The results were compared with those from 13 age matched patients with Parkinson's disease and six healthy volunteers.

Results-In all but one patient with progressive supranuclear palsy, there was a distinct difference in muscle tonus between the neck and trunk. A tonic shortening reaction characteristic of dystonia and an increased tonic stretch reflex (rigidity) were present in the neck muscles of patients with progressive supranuclear palsy, whereas only normal to moderately increased tonus was noted in the truncal muscles (neck $v$ trunk, shortening reaction $p=0.0001$; stretch reflex $p=0.0241$ ). Follow up studies disclosed an increase in axial muscle tonus with predilection for the neck in three of four patients. In the $\mathbf{1 3}$ patients with Parkinson's disease, however, no significant difference was found in muscle rigidity between the neck and trunk.
\end{abstract}

Conclusion-Mild changes in truncal muscle tonus with prominent neck dystonia and rigidity are characteristic of progressive supranuclear palsy. It is suggested that separate clinical evaluation of muscle tonus in the neck and trunk may be helpful for distinguishing progressive supranuclear palsy from Parkinson's disease.

\section{(F Neurol Neurosurg Psychiatry 1998;64:190-196)}

Keywords: progressive supranuclear palsy; Parkinson's disease; truncal muscle tonus; surface electromyography

Dr Atsushi Komiyama, Department of Neurology, Urafune Hospital, Yokohama City University, 3-46 Urafune-cho, Minami-ku, Yokohama 232, Japan. Telephone 008145253 5381; fax 0081452537346 .

Received 29 November 1996 and in revised form

7 May 1997

Accepted 29 July 1997 remains unclear, presumably because the methodology for clinical evaluation of truncal tonus has not been established. In addition, neck and truncal muscles are usually treated as one unit of axial muscles. ${ }^{1-7}$ Although factors contributing to neck muscle hypertonus have been described clinically as rigidity, ${ }^{45}$ dystonic rigidity, ${ }^{12}$ or rigidity and dystonia, ${ }^{3}{ }^{3}$ no objective study has been performed.

Recently, Nagumo and Hirayama ${ }^{8}$ developed a bedside technique to evaluate truncal muscle tonus, and they showed truncal rigidity in Parkinson's disease. In the present study, we examined muscle tonus of the neck and trunk separately in patients with progressive supranuclear palsy by means of clinical assessment and surface EMG to determine the character and distribution of abnormal muscle tonus in the body axis.

\section{Subjects and methods}

SUBJECTS

Thirteen patients, seven men and six women, with well characterised progressive supranuclear palsy ${ }^{1-7}$ served as the primary study group (mean age (SD), 65.2 (7.0) years). Eleven of these were outpatients from the Department of Neurology, Yokohama City University School of Medicine, Yokohama, and the two others were outpatients from the Department of Psychiatry, Nanasawa Rehabilitation Center, Atsugi. The duration of the progressive supranuclear palsy symptoms ranged from one to seven years (mean duration (SD), 3.6 (1.6) years). Motor symptoms were advanced, with five patients at stage 3 , five at stage 4 , and three at stage 5 (mean (SD), $3.8(0.8)$ ) as defined by the Hoehn and Yahr scale. ${ }^{9}$ All the patients were rated at stage 3 or greater, because they showed prominent postural instability with frequent falls. At the time of testing, seven patients were taking carbidopa-levodopa in combination with amantadine or droxydopa, three patients were taking amitryptiline, and three were not taking any medication.

Thirteen patients with typical clinical features of Parkinson's disease, eight men and five women (mean age (SD), 66.0 (6.1) years), and six healthy subjects, three men and three women, with no apparent neurological abnormality (mean age (SD), 62.1 (7.3) years) served as a comparison group. All patients with Parkinson's disease were outpatients from the Department of Neurology, Yokohama City University School of Medicine, Yokohama. The duration of the Parkinson's disease symptoms ranged from two to 13 years (mean duration (SD), 5.1 (3.6) years). Despite the longer duration of their disease, the patients with Parkinson's disease showed motor dysfunction 

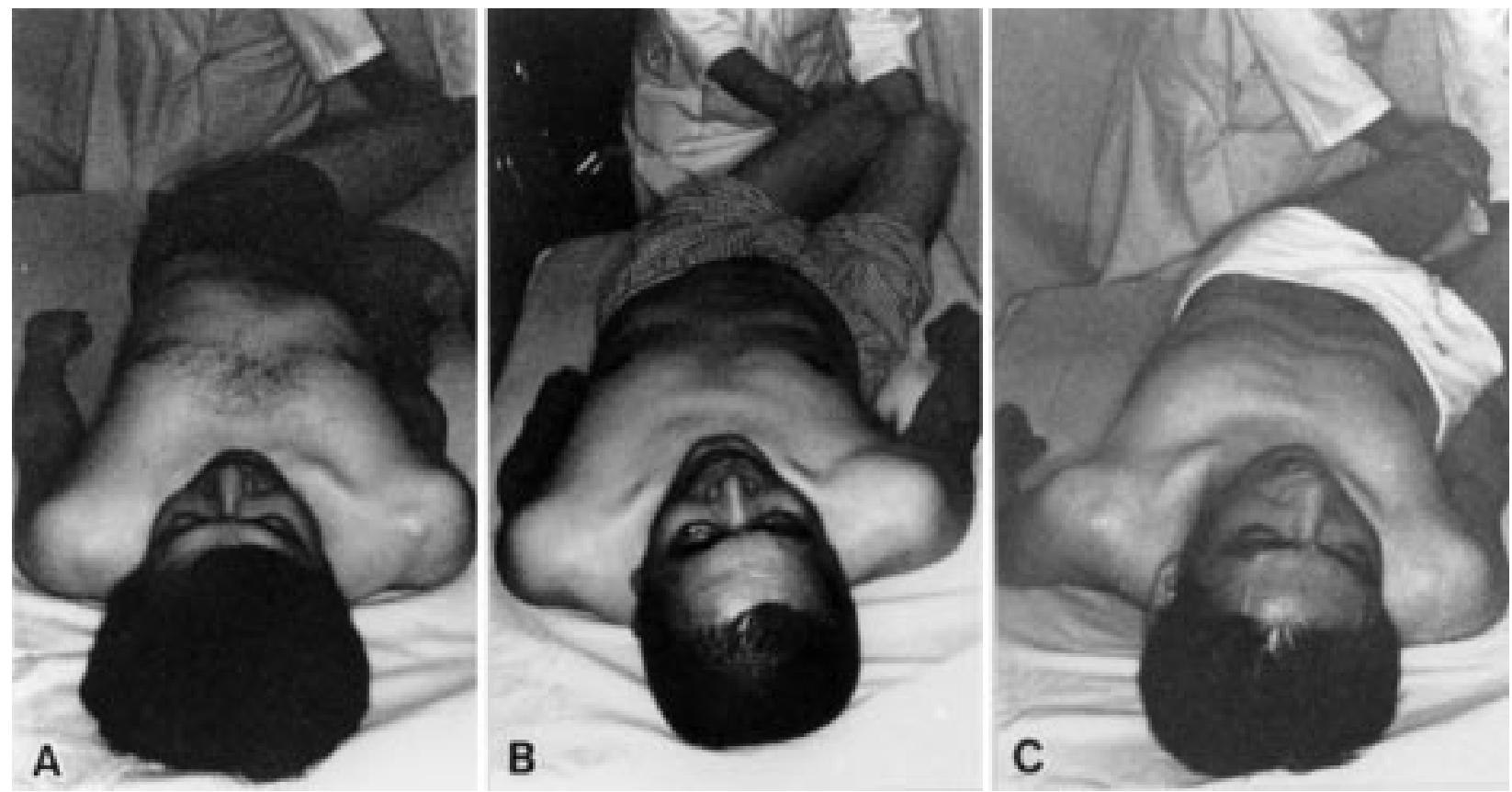

Figure 1 Evaluation of truncal muscle tonus by the alternating knee tilt method. (A) A healthy volunteer aged 33. This picture is provided to show the method and normal response of the evaluation. (B) $A 61$ year old man (patient 9) with progressive supranuclear palsy. (C) $A 55$ year old man (patient 1 ) with Parkinson's disease. Passive tilting of the flexed knees of the healthy volunteer and the patient with progressive supranuclear palsy do not produce resistance of the truncal muscles $(A, B)$. In the patient with Parkinson's disease resistance of the truncal muscles is pronounced and results in the raising of the contralateral trunk and shoulder $(C)$.

which was milder than that of the patients with progressive supranuclear palsy, with five patients at stage 2 , five at stage 3 , and three at stage 4 (mean (SD), $2.8(0.8)$ ) on the Hoehn and Yahr scale. However, the difference in clinical severity was unlikely to have influenced the results of this study as the patients with Parkinson's disease evidenced more increased tonus of the truncal muscle than the patients with progressive supranuclear palsy. At the time of testing, all 13 patients were taking carbidopa-levodopa in combination with bromocriptine, amantadine, and anticholinergic drugs at varying doses. Patients with Parkinson's disease or progressive supranuclear palsy were tested one to two hours after medication; all the patients with Parkinson's disease were in an "on" period, when the tests were performed. No "on" period dyskinesia of the neck or trunk was noted in any patients with Parkinson's disease.

Although the presence of musculoskeletal diseases in the hip and lumbar spine may influence the results of the truncal muscle testing, there were no patients with such diseases causing discomfort and secondary muscle contraction.

MUSCLE TONUS EVALUATION AND SURFACE EMG RECORDINGS

Under the conditions described below, the subjects were instructed to attempt to rotate their head or trunk slowly at a constant speed during voluntary contraction, and to completely relax the axial muscles tested during passive rotation. Special care was used during passive manipulation to eliminate contamination from voluntary movements that may simulate a tonic shortening reaction in surface EMG recordings. When muscle discharges were recorded in agonist muscles without clinical evidence of hypertonus, such recordings were considered as voluntary contractions and excluded from the study. All tests were conducted in an air conditioned room at $24-26^{\circ} \mathrm{C}$

Neck muscle

To evaluate neck muscle tonus, the subjects were examined at rest and with voluntary or passive rotating movements of the head during sitting. ${ }^{10}$ The surface electrodes (NM 3125, Nihonkoden, Japan) were placed in pairs over the sternocleidomastoid muscles and splenius muscles on both sides; a potentiometer was fixed on top of the head to determine the angle of neck rotation. EMG activities ranging from $20 \mathrm{~Hz}$ to $1 \mathrm{kHz}$ were amplified and recorded with a Neuropack 8 (Nihonkoden, Japan). We also compared neck muscle tonus between the sitting and supine positions to examine any postural influences.

\section{Truncal muscle}

Using the alternating knee tilt method described by Nagumo and Hirayama, ${ }^{8}$ truncal tonus of the subjects was assessed in the supine position. The body axis was rotated voluntarily by the subject or passively by the examiner. With the knees flexed, the legs were supported and alternately tilted rightward and leftward (fig 1A). Surface electrodes were placed symmetrically in pairs over the thoracic erector spinae muscles and obliquus internus abdominis muscles. The thoracic portions of the erector spinae muscles were examined because these levels have previously been shown to be the primary source of the truncal rigidity in Parkinson's disease. ${ }^{8}$ Surface electrodes were placed over the most prominent parts of the 
Summary of clinical evaluation and surface electromyographic recordings during passive movements

\begin{tabular}{|c|c|c|c|c|c|c|c|}
\hline \multirow[b]{3}{*}{ Patient no } & \multirow[b]{3}{*}{ Age/ sex } & \multirow[b]{3}{*}{ Severity* } & \multirow[b]{3}{*}{$\begin{array}{l}\text { Disease } \\
\text { duration }(y)\end{array}$} & \multicolumn{4}{|c|}{ Muscle hypertonicity } \\
\hline & & & & \multicolumn{2}{|l|}{ Neck } & \multicolumn{2}{|l|}{ Trunk } \\
\hline & & & & $\begin{array}{l}\text { Shortening } \\
\text { reaction }\end{array}$ & $\begin{array}{l}\text { Stretch } \\
\text { reflex }\end{array}$ & $\begin{array}{l}\text { Shortening } \\
\text { reaction }\end{array}$ & $\begin{array}{l}\text { Stretch } \\
\text { reflex }\end{array}$ \\
\hline \multicolumn{8}{|c|}{ Progressive supranuclear palsy (PSP): } \\
\hline \multirow{2}{*}{1} & $79 / \mathrm{M}$ & 5 & 6 & 2 & 1 & 1 & 1 \\
\hline & 80 & 5 & 7 & 3 & 1 & 2 & 2 \\
\hline 2 & $68 / \mathrm{M}$ & 5 & 4 & 2 & 3 & 1 & 1 \\
\hline 3 & $61 / \mathrm{F}$ & 5 & 4 & 3 & 2 & 0 & 1 \\
\hline 4 & $74 / \mathrm{M}$ & 4 & 4 & 2 & 0 & 1 & 0 \\
\hline 5 & $64 / \mathrm{F}$ & 4 & 4 & 2 & 1 & 1 & 0 \\
\hline 6 & $52 / \mathrm{F}$ & 4 & 4 & 1 & 2 & 1 & 1 \\
\hline \multirow[t]{2}{*}{7} & $60 / \mathrm{M}$ & 4 & 2 & 2 & 1 & 0 & 0 \\
\hline & 63 & 5 & 5 & 2 & 3 & 1 & 0 \\
\hline 8 & $66 / \mathrm{F}$ & 4 & 2 & 1 & 1 & 0 & 0 \\
\hline \multirow[t]{2}{*}{9} & $61 / \mathrm{M}$ & 3 & 7 & 3 & 1 & $0 \dagger$ & 0 \\
\hline & 63 & 3 & 9 & 3 & 1 & 2 & 2 \\
\hline 10 & $68 / \mathrm{F}$ & 3 & 3 & 3 & 1 & 1 & 0 \\
\hline \multirow[t]{2}{*}{11} & $70 / \mathrm{F}$ & 3 & 3 & 2 & 1 & 1 & 0 \\
\hline & 73 & 5 & 6 & 2 & 3 & 1 & 1 \\
\hline 12 & $59 / \mathrm{M}$ & 3 & 3 & 2 & 0 & 0 & 1 \\
\hline 13 & $66 / \mathrm{M}$ & 3 & 1 & 1 & 0 & 0 & 0 \\
\hline \multicolumn{8}{|c|}{ Parkinson's disease (PD): } \\
\hline 1 & $55 / \mathrm{M}$ & 4 & 13 & 1 & 3 & 3 & 3 \\
\hline 2 & $71 / \mathrm{F}$ & 4 & 10 & 1 & 2 & 1 & 2 \\
\hline 3 & $78 / \mathrm{M}$ & 4 & 2 & 2 & 2 & 2 & 2 \\
\hline 4 & $60 / \mathrm{M}$ & 3 & 10 & 2 & 1 & 2 & 2 \\
\hline 5 & $70 / \mathrm{F}$ & 3 & 5 & 2 & 2 & 2 & 1 \\
\hline 6 & $65 / \mathrm{F}$ & 3 & 4 & 2 & 1 & 3 & 1 \\
\hline 7 & $69 / \mathrm{M}$ & 3 & 4 & 1 & 1 & 2 & 1 \\
\hline 8 & $61 / \mathrm{M}$ & 3 & 4 & 1 & 1 & 0 & 1 \\
\hline 9 & $62 / \mathrm{M}$ & 2 & 5 & 2 & 1 & 2 & 2 \\
\hline 10 & $69 / \mathrm{M}$ & 2 & 3 & 0 & 1 & 1 & 1 \\
\hline 11 & $62 / \mathrm{M}$ & 2 & 2 & 1 & 1 & 2 & 2 \\
\hline 12 & $65 / \mathrm{F}$ & 2 & 2 & 0 & 1 & 1 & 1 \\
\hline 13 & $71 / \mathrm{F}$ & 2 & 2 & 1 & 1 & 1 & 1 \\
\hline \multicolumn{8}{|c|}{ Healthy volunteers: } \\
\hline 1 & $56 / \mathrm{F}$ & & & 0 & 0 & 0 & 0 \\
\hline 2 & $59 / \mathrm{F}$ & & & $0 \dagger$ & 0 & 0 & 0 \\
\hline 3 & $48 / \mathrm{F}$ & & & $0 \dagger$ & 0 & 0 & 0 \\
\hline 4 & $76 / \mathrm{M}$ & & & 0 & 0 & 0 & 0 \\
\hline 5 & $64 / \mathrm{M}$ & & & 0 & 0 & 0 & 0 \\
\hline 6 & $68 / \mathrm{M}$ & & & 0 & 0 & 0 & 0 \\
\hline
\end{tabular}

${ }^{\star}$ Hoehn and Yahr scale; $0=$ hypertonus absent; $1=$ mild; $2=$ moderate; $3=$ severe.

†Graded as 0 because surface EMG activity is not accompanied by clinical evidence of muscle hypertonus. whole trunk because of the pronounced increase in the truncal muscle tonus). The grade of the muscle hypertonus has been determined by that of the most severe part of the axial muscles tested. Surface EMG was used to confirm the type of muscle hypertonus - that is, increase in the tonic stretch reflex or tonic shortening reaction, and also to define the grade of the coexistent muscle hypertonus with lesser EMG activities. When clinical evaluation and surface EMG studies indicated severe resistance and an increase in the tonic shortening reaction as a main abnormality, respectively, the patient was considered to have muscle shortening of grade 3. The surface EMG may also show an increase in the tonic stretch reflex; up to two thirds and one third of the amplitude of grade 3 were arbitrarily defined as grades 2 and 1 . When the level of the tonic shortening reaction was grade 2 and the amplitude of EMG indicating the tonic stretch reflex was more or less than half of that of the shortening, the coexistent tonic stretch reflex was assumed to be grade 2 or 1 . Finally, in patients with clinical hypertonicity of grade 1, any increased EMG activity was judged to be grade 1 . In patients exhibiting severe hypertonus, clinical manipulation of the neck resulted in low amplitude, poorly reproducible rotations due to the pronounced resistance during passive movement (patients 7 and 11 with progressive supranuclear palsy at the second evaluation). It is conceivable that when the truncal hypertonus increases so much that the whole body axis rotates with raising of the hip, surface EMG activity may decrease paradoxically, but there were no such patients in this study.

STATISTICAL ANALYSIS

Group means were compared by unpaired, two tailed Mann-Whitney test. A p value $<0.05$ was considered to be significant. Correlation between two groups was carried out with Spearman rank correlation. spinous process at the T3-4 and T8-9 levels." Surface electrodes were fixed over the obliquus internus abdominis $3 \mathrm{~cm}$ above and perpendicular to the inguinal ligaments and $4 \mathrm{~cm}$ medial to the anterior superior iliac spine. ${ }^{12}$ To monitor any change in knee position, a fluorescent ball on the kneecap was traced by a camera and recorded with a Neuropack 8 . Knee tilt toward the right side of the subject's body was defined as rightward rotation in this study.

\section{Muscle hypertonus grading}

Our attempt to normalise the surface EMG as a percentage of voluntary contraction was hampered by reduced or no voluntary axial movements with disease progression. Therefore, the level of muscle hypertonus was clinically graded according to a four point scale (comments applied to the assessment of the truncal muscle tonus): $0=$ resistance absent (no raising of the shoulder); $1=$ mild resistance (with or without slight raising of the shoulder); $2=$ moderate resistance with full range of motion (with apparent raising of the shoulder); $3=$ severe resistance, range of motion achieved with difficulty or not achieved (rotation of the

\section{Results}

In the healthy subjects, voluntary neck rotation while sitting caused continuous and decremental discharges in the agonist muscles-that is, the left sternocleidomastoid and right splenius muscles during rightward rotation. However, with passive rotation of the neck, only a minimal discharge was elicited. Passive rotation of the neck in a supine position was often complicated by voluntary contraction of the sternocleidomastoid muscles regardless of the presence of a pillow or its height. Voluntary trunk rotation gave rise to discharges in agonist muscles-for example, left thoracic erector spinae and left obliquus internus abdominis muscles during rightward rotation, whereas passive rotation produced no resistance (fig 1A), thereby generating no appreciable levels of EMG discharges.

In the patients with progressive supranuclear palsy, except for patient 13 who showed only slight changes in neck muscles, there were apparent discrepancies in muscle tonus between the neck and trunk. Voluntary neck 


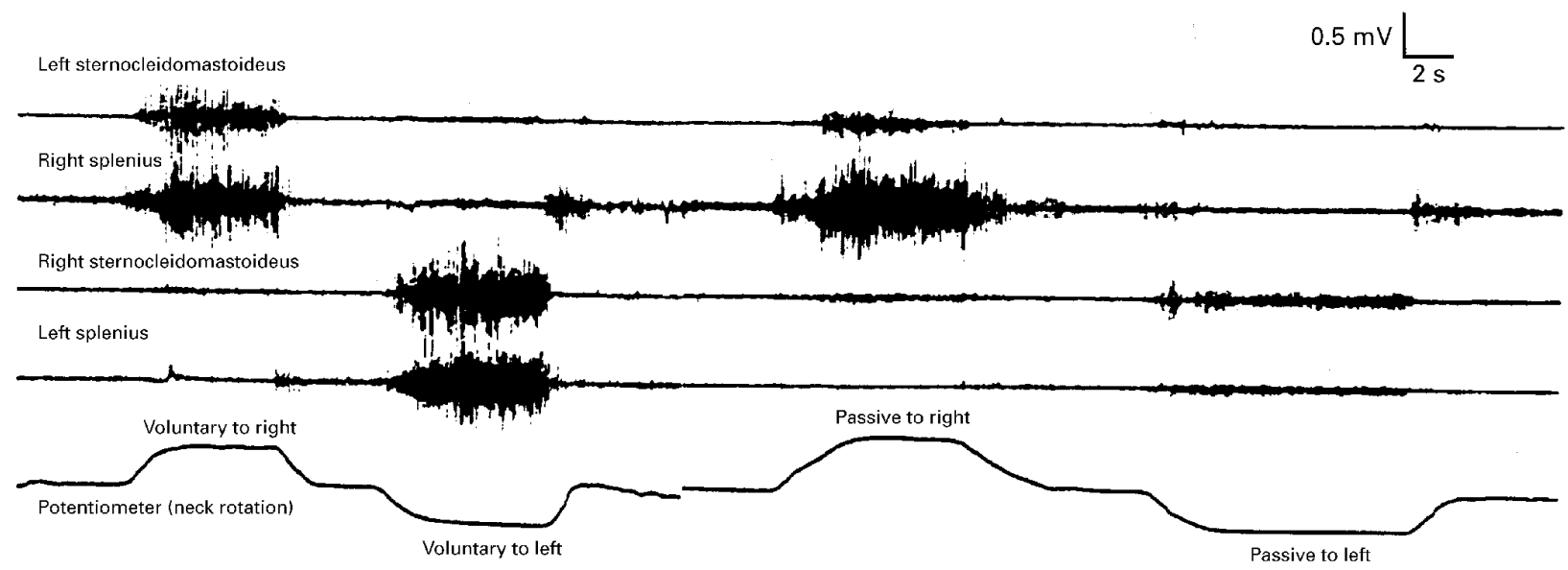

A

Left obliquus internus abdominis

\section{B}

Figure 2 Representative surface EMG recordings from the neck $(A)$ and trunk (B) during voluntary (in the first half of the trace) and passive (in the second half of the trace) rotation in a 61 year old man (patient 9) with progressive supranuclear palsy. Voluntary rotation of the neck produced a near normal discharge pattern except for minimal discharges from the right sternocleidomastoid and left splenius muscles during leftward rotation. Passive rightward rotation caused prominent EMG discharges in the right splenius and, to a lesser degree, in the left sternocleidomastoid muscles (a tonic shortening reaction). Contralateral leftward rotation also evoked a slight discharge in the right sternocleidomastoid and left splenius muscles. In addition, a slight discharge was detectable over antagonist muscles (a tonic stretch reflex). Voluntary rotation of the trunk produced normal discharges from the contralateral agonist muscles. Passive rotation of the trunk disclosed normal tonus except for mild discharges from the right T3 erector spinae muscle during leftward rotation. Because of absence of clinical hypertonus, the EMG discharges are considered as voluntary contraction and excluded from the study.

rotation caused continuous discharges in agonist muscles as in healthy subjects. However, simultaneous contraction of antagonist muscles (impairment of reciprocal innervation) was also detected in all patients with progressive supranuclear palsy except for patient 13 (fig $2 \mathrm{~A})$. Voluntary rotation of the axial muscles was impossible in two patients $(2,3)$. When the neck was rotated passively, a tonic shortening reaction consistent with dystonia ${ }^{10}{ }^{13}$ was detected in all patients with progressive supranuclear palsy with passive neck rotation producing discharges in agonist muscles (table; fig $2 \mathrm{~A})$. In addition, antagonist muscle contraction, usually to a lesser degree, was seen in 12 patients, which indicates an increase in the tonic stretch reflex characteristic of rigidity. ${ }^{13}$ Neck muscle tonus did not decrease in the supine position as it was often complicated by ongoing background contraction of the sternocleidomastoid muscles (fig 3). In seven patients
$(1,3,5,7,8,9,11)$, an upturned head was clinically recognisable. Truncal muscles, by contrast, showed normal to moderately increased tonus. Voluntary rotation of the trunk produced a normal pattern of EMG discharges over agonist muscles, and passive tilting of the flexed knees caused milder muscle discharges than passive neck rotation (shortening reaction $p=0.0001$, stretch reflex $p=0.0241$; table, fig $1 \mathrm{~B}$ and fig $2 \mathrm{~B})$. In three patients $(3,6,7)$, some differences were detectable in truncal tonus between the right and left side.

Evaluation of the axial muscles during follow up visits disclosed an increase in muscle tonus with predilection for the neck in three of four patients; moderate truncal rigidity and dystonia developed in patient 9 after two years (table). At the second examination, voluntary rotation of the axial muscles on command was lost in patients 7 and 11 . In addition, passive rotation of the neck had become almost impossible in 


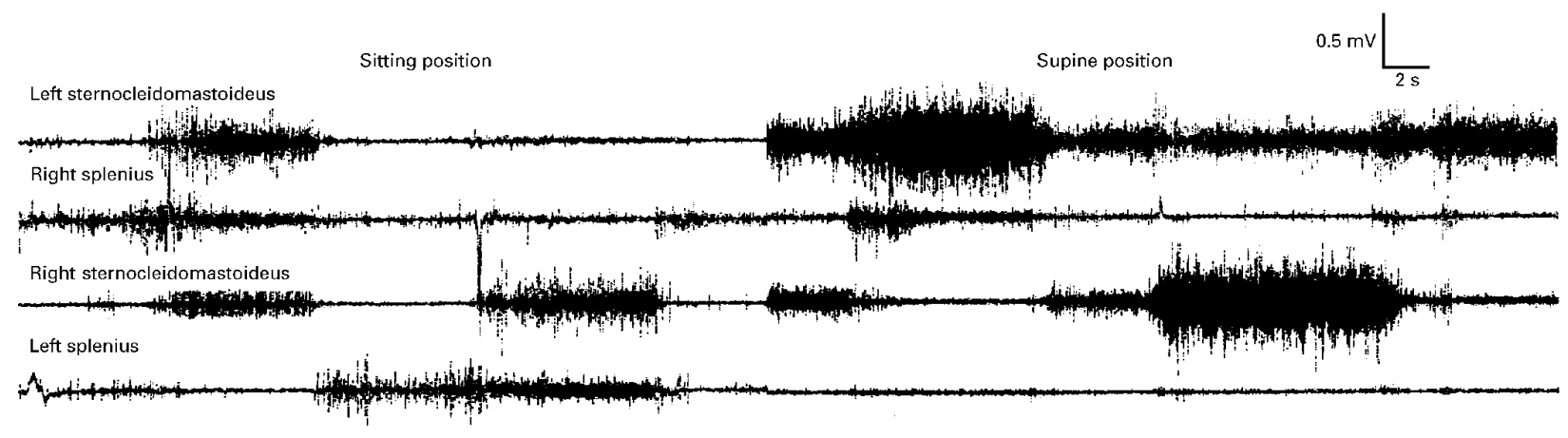

Potentiometer (neck rotation)

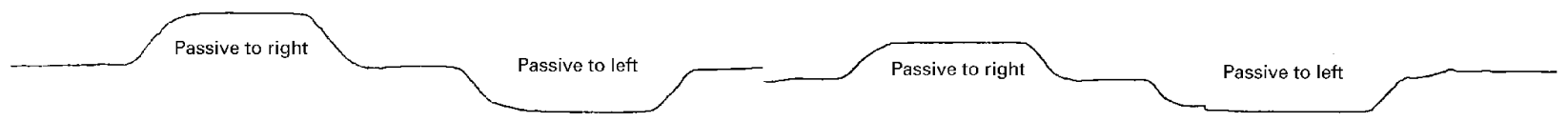

Figure 3 Surface EMG recordings from the neck muscles during passive rotation in the sitting and supine position in patient 9 with progressive supranuclear palsy. Note the background contamination from sternocleidomastoid muscle activity in the supine position, although there was no consistent decrease in the neck muscle tonus during the supine position.

these two patients as a result of the severe muscle hypertonus associated with contractures, whereas passive rotation of the trunk showed mildly increased tonus.

In the patients with Parkinson's disease, when rotated voluntarily, muscle discharges were found in antagonist muscles as well as agonist muscles (fig 4). This loss of reciprocal innervation hampered normal neck or truncal movement. Passive rotation of neck or truncal muscles caused an increase not only in the tonic stretch reflex but also in the tonic shortening reaction ${ }^{13}$ (table; fig $1 \mathrm{C}$, fig 4 ). There was no difference between the neck and trunk muscle tonus (shortening reaction $\mathrm{p}=0.4739$, stretch reflex $\mathrm{p}=0.1601$; table). However, significant correlation was found in the shortening reaction only (shortening reaction $r=0.583, \mathrm{p}=0.0434$; stretch reflex $r=0.502$, $\mathrm{p}=0.0820$ ).

When patients with progressive supranuclear palsy and those with Parkinson's disease were compared, the neck shortening was significantly increased in progressive supranuclear palsy $(\mathrm{p}=0.0174)$, whereas the truncal shortening reaction $(\mathrm{p}=0.0002)$ and stretch reflex $(p=0.0019)$ were increased in Parkinson's disease.

\section{Discussion}

The sternocleidomastoid and splenius muscles are the major rotators of the neck. The first rotates the neck to the contralateral side and the second to the ipsilateral side. ${ }^{10}$ Rotation of the trunk involves the contralateral erector spinae and obliquus internus abdominis muscles. ${ }^{8}$ In healthy people, voluntary rotation produces continuous discharges over these agonist muscles, whereas little muscle discharge occurs during passive rotation. Discharges from agonist muscles represent muscle shortening during passive rotation and indicate the presence of a tonic shortening reaction, a finding associated with dystonia. ${ }^{10} 13$ Other conditions, including spasticity, athetosis, and painful inflammatory musculoskeletal diseases, can cause a tonic shortening reaction, ${ }^{13}$ but did not play a major part in our patients. On the other hand, when muscle discharges are present in stretched antagonist muscles, they reflect an increase in the tonic stretch reflex, a finding characteristic of rigidity. ${ }^{13}$

Our present study showed a distinct difference in muscle tonus between the neck and trunk in all but one patient with progressive supranuclear palsy. Apparent dystonia with a component of rigidity was found in the neck muscles, but only normal to moderately increased tonus was noted in the truncal muscles. In the patients with Parkinson's disease, however, the axial tonus testing during "on" periods showed that truncal rigidity with a component of dystonia ${ }^{13}$ occasionally exceeded that of neck muscles. ${ }^{8}$ These findings are essentially consistent with the latest report by Nagumo and Hirayama, ${ }^{14}$ although their study is solely based on the clinical assessment. Steele et $a l^{1}$ in their initial description of progressive supranuclear palsy reported that dystonic rigidity of the neck and upper trunk is a common feature of progressive supranuclear palsy and that a more widespread hypertonicity involving the limbs develops at the later stage of the disease. Barr ${ }^{2}$ stated that the upper trunk is initially involved, followed by the lower axial musculature. However, although longer duration of disease was associated with pronounced neck dystonia and rigidity, truncal muscle tonus remained relatively mild in our patients. Because of a lack of surface EMG evaluation of four limbs, the present study failed to delineate the relation between the axial (neck and trunk) and limb muscle tonus. In contrast to the cases of progressive supranuclear palsy, Nagumo and Hirayama ${ }^{14}$ showed apparent correlation in the rigidity between the axial and limb muscles in Parkinson's disease. Interestingly, they noted that when the limb rigidity was predominant on one side, the truncal rigidity was severer on the opposite side. ${ }^{14}$

To our knowledge, the pathophysiological mechanism for this discrepancy in axial muscle tonus in progressive supranuclear palsy has never been considered. Behrman et $a l^{15}$ have postulated that neck dystonia in progressive supranuclear palsy represents a form of 


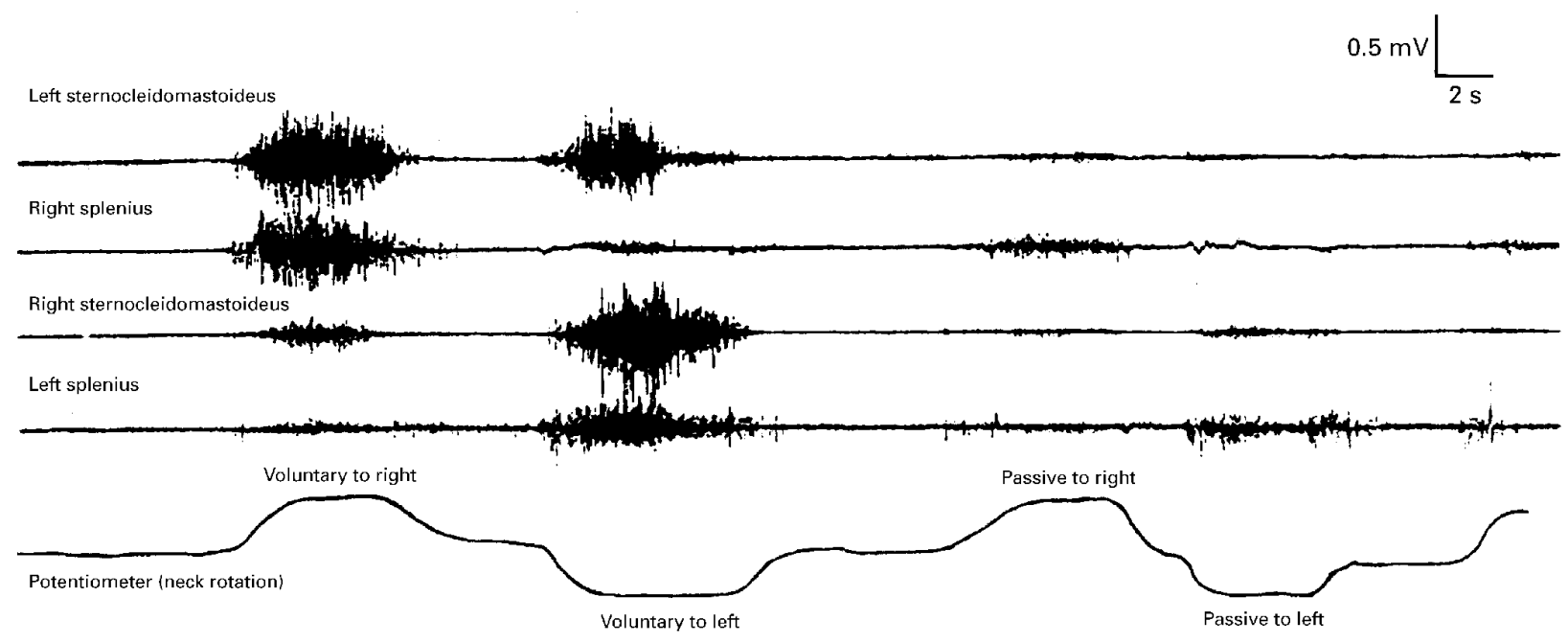

A

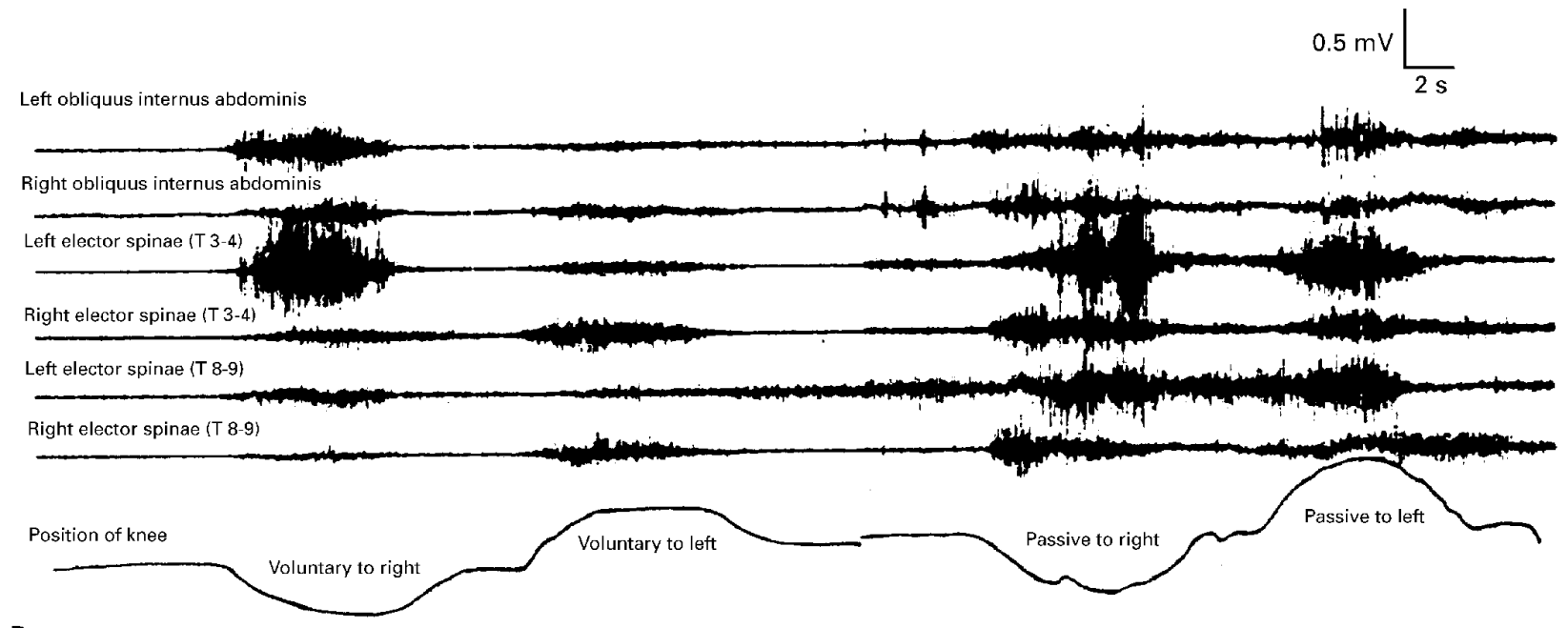

B

Figure 4 Representative surface EMG recordings from the neck (A) and trunk (B) during voluntary (in the first half of the trace) and passive (in the second half of the trace) movements in a 62 year old man (patient 11) with Parkinson's disease. In addition to the normal pattern of discharges in agonist muscles, voluntary rotation of the neck produced discharges from the antagonist muscles. Passive rotation of the neck caused mild discharges in both agonist and antagonist muscles. Voluntary rotation of the trunk evoked discharges both in agonist and antagonist muscles, impeding normal movements. Passive rotation of the trunk produced prominent discharges from erector spinae and obliquus internus abdominis muscles bilaterally, indicating the coexistence of increases in the tonic stretch reflex and shortening reaction.

decerebrate posture due to a mesencephalic lesion, which is also responsible for supranuclear vertical gaze palsy. An abnormality in the rostral brainstem and diencephalon, the regions maximally affected in progressive supranuclear palsy, have also been implicated in the pathogenesis of dystonia. ${ }^{6}$ Recent studies with cats showed that the characteristic upturned head and impairment of vertical eye movement were produced by bilateral kainic acid lesions of the interstitial nucleus of Cajal. ${ }^{16}$ In addition, a literature review of necropsied cases of progressive supranuclear palsy disclosed that nerve cell loss was found in the interstitial nucleus of Cajal in patients who had developed dorsiflexion of the neck. ${ }^{17}$ Therefore, involvement of the interstitial nucleus of Cajal is likely to account for not only the discrepancy in the axial muscle tonus but also the dystonic nature of the neck muscles in progressive supranuclear palsy.
In our study, patient 9 was originally diagnosed and treated for Parkinson's disease. Despite the original comments of Steele et al that there should be little confusion between progressive supranuclear palsy and Parkinson's disease, patients with progressive supranuclear palsy can sometimes present with a bradykinetic rigid syndrome and may be misdiagnosed as having Parkinson's disease. ${ }^{256}$ Without a diagnostic test or specific biological marker, the diagnosis of progressive supranuclear palsy remains subjective. The discrepancy in muscle tonus between the neck and trunk is readily apparent in patients with progressive supranuclear palsy except in the early stages when the neck muscle tonus remains unchanged. In addition to the characteristic abnormality of eye movement and postural instability with frequent falls, ${ }^{1-7}$ a mild increase in truncal tonus with prominent neck dystonia and rigidity may help differentiate progressive supranuclear 
palsy from Parkinson's disease. However, the patients with progressive supranuclear palsy who have relatively preserved eye movements would represent the greatest difficulty in the differential diagnosis, because such patients have less mesencephalic involvement, and therefore, would probably have less neck muscle hypertonus ${ }^{2}$ and less discrepancy between the neck and truncal tonus.

We thank Drs T Takahashi and H Nagatomo, Nanasawa Rehabilitation Center for referral of patients with progressive supranuclear palsy, Dr T Mano, Nagoya University for reading the manuscript, and the assessors for valuable comments and criticisms.

1 Steele JC, Richardson JC, Olszewski J. Progressive supranuclear palsy. A heterogeneous degeneration involving the brain stem, basal ganglia and cerebellum with vertical gaze and pseudobulbar palsy, nuchal dystonia and dementia. Arch Neurol 1964;10:333-59.

2 Barr AN. Progressive supranuclear palsy. In: Vinken PJ Bruyn GW, Klawans HL, eds. Handbook of clinical neurology. Vol 49. Amsterdam: Elsevier, 1986:239-54.

3 Lees AJ. The Steele-Richardson-Olszewski syndrome (progressive supranuclear palsy). In: Marsden CD, Fahn S, eds Movement disorders 2. London: Butterworth 1987:272-87.

4 Golbe LI, Davis PH. Progressive supranuclear palsy. Recent advances. In: Jankovic J, Tolosa E, eds. Parkinson's disease berg, 1988:121-30.

5 Weiner WJ, Lang AE. Movement disorders. A comprehensive survey. Mount Kisco: Futura, 1989:147-61.

6 Jankovic J, Friedman DI, Pirozzolo FJ, et al. Progressive Jankovic J, Friedman DI, Pirozzolo FJ, et al. Progressive supranuclear palsy: motor, neurobehavioral, and neuro-
ophthalmic findings. In: Streifler MB, Korczyn AD, Mela- med E, et al, eds. Advances in neurology. Vol 53. Parkinson's disease: anatomy, pathology, and therapy. New York: Raven Press, 1990:293-304.

7 Duvoisin RC. Clinical diagnosis. In: Litvan I, Agid Y, eds. Progressive supranuclear palsy. Clinical and research approaches. New York: Oxford University Press, 1992:15-33.

8 Nagumo K, Hirayama K. A study on truncal rigidity in Parkinsonism. Evaluation of diagnostic test and electrophysiological study. Clin Neurol 1993:33:27-35. (In Japanese with English abstract.)

9 Hoehn MM, Yahr MD. Parkinsonism: onset, progression, Hoehn MM, Yahr MD. Parkinsonism: onset
and mortality. Neurology 1967;17:427-42.

10 Hasegawa O. Spasmodic torticollis: its electromyographic recording and treatment by alcoholization. In: Berthoz A, Graf W, Vidal PP, eds. The head-neck sensory motor system. New York: Oxford University Press, 1992:659-62.

11 Floyd WF, Silver PHS. The function of the erectores spinae muscles in certain movements and postures in man. $\mathcal{f}$ Physiol 1955;129:184-203.

12 Waters RL, Morris JM. Electrical activity of muscles of the trunk during walking. F Anat 1972;111:191-9.

13 Bathien N, Rondot P. Assessment of motor functions in extrapyramidal disorders. In: Eccles D, ed. Recent achievements in restorative neurology 1: upper motor neuron functions and dysfunctions. Bazel: Karger 1985:211-21.

14 Nagumo K, Hirayama K. Axial (neck and trunk) rigidity in Parkinson's disease, striatonigral degeneration and proressive supranuclear palsy. Clin Neurol 1996:36:1129-35. (In Japanese with English abstract.)

15 Behrman S, Carroll JD, Janota I, et al. Progressive supranuclear palsy. Clinico-pathological study of four cases. Brain 969;92:663-78.

6 Fukushima K, Fukushima J, Terashima T. The pathways responsible for the characteristic head posture produced by lesions of the interstitial nucleus of Cajal in the cat. Exp Brain Res 1987;68:88-102.

17 Fukushima-Kudo J, Fukushima K, Tashiro K. Rigidity and dorsiflexion of the neck in progressive supranuclear palsy and interstitial nucleus of Cajal. $f$ Neurol Neurosurg and interstitial nucleus of 